\title{
NTT SPECTRA OF THE FLARE STARS HM1 AND HM2 IN THE R CORONAE AUSTRINAE AGGREGATE
}

\author{
H. W. DUERBECK ${ }^{1,2}$ and M. K. TSVETKOV $2,3,4$ \\ ${ }^{1}$ European Southern Observatory, La Silla, Chile \\ ${ }^{2}$ Astronomisches Institut der Universität Münster, F.R. Germany \\ ${ }^{3}$ Department of Astronomy and National Astronomical Observatory, \\ Bulgarian Academy of Sciences, Sofia, Bulgaria \\ ${ }^{4}$ Research Fellow, Alexander von Humboldt Foundation, Bonn-Bad Godesberg.
}

Abstract. Spectroscopic observations of the flare stars HM1 and HM2 (V667 CrA) in the direction of the $\mathbf{R}$ Coronae Austrinae aggregate are presented. The respective spectral classes are $\mathrm{dM} 1 \mathrm{e}$ and $\mathrm{dM} 6 \mathrm{e}$. Both stars are at the distance $40 \mathrm{pc}$, much closer than the $\mathrm{R}$ CrA association.

The $\mathrm{R}$ CrA region is a well-known star forming region, investigated by a variety of observational techniques. The interest in the region is due to the presence of the $\mathrm{R} \mathrm{CrA}$ $\mathrm{T}$ association, containing the bright reflection nebulae NGC 6726 and NGC 6729, Herbig $\mathrm{Ae} / \mathrm{Be}$ stars, several well established T Tau type stars, $\mathrm{H} \alpha$ emission stars, two Herbig-Haro objects and many rapid irregular variables of unknown type. The distance to the $\mathrm{R} \mathrm{CrA}$ dark cloud is about $130 \mathrm{pc}$, according to Marraco and Rydgren (1981).

Among the young stellar objects two flare stars were discovered by Hardy and Mendoza (1968) on plates taken with the Curtis Schmidt telescope. During multi-exposure observations in September 1968, the ultraviolet brightness of both stars increased by two magnitudes in less than two hours. The data of the stars and flare events are summarized in Table 1.

TABLE 1. Data of the flare stars HM1 and HM2

\begin{tabular}{|c|c|c|c|c|c|c|c|}
\hline $\begin{array}{c}\text { Star } \\
\text { design. }\end{array}$ & $\begin{array}{l}\text { GCVS } \\
\text { design. }\end{array}$ & $\begin{array}{c}\mathrm{RA} \\
(1950)\end{array}$ & $\begin{array}{c}\text { Dec } \\
(1950)\end{array}$ & $\begin{array}{c}\mathrm{JD}(\max ) \\
2440114 \ldots\end{array}$ & $\begin{array}{c}\operatorname{mag} \\
\mathrm{pg}\end{array}$ & $\begin{array}{l}\text { Duration } \\
\min \end{array}$ & $\begin{array}{l}\text { Sp. } \\
\text { type }\end{array}$ \\
\hline HM1 & Anon & $18^{\mathrm{h}} 54^{\mathrm{m}} 08^{\mathrm{s}}$ & $-36^{\circ} 38^{\prime} .6$ & 0.518 & $13^{\mathrm{m}} \cdot 6$ & 90 & $\mathrm{dM} 1 \mathrm{e}$ \\
\hline HM2 & V667 CrA & $\begin{array}{lll}18 & 57 & 54\end{array}$ & $\begin{array}{lll}-37 & 00 & .7\end{array}$ & 0.550 & 18 & 60 & $\mathrm{dM} 6 \mathrm{e}$ \\
\hline
\end{tabular}

The spectra of both stars were taken on 1989 September 8 with the Faint Object Spectrograph and Camera EFOSC 2, attached to the $3.5 \mathrm{~m}$ New Technology Telescope of ESO. Exposure times in three different spectral regions were of the order of a few minutes each. 
The spectra were flatfielded, wavelength- and flux-calibrated by means of standard objects (lamp, planetary nebula, spectrophotometric standard), and combined into one spectrum from three (HM 1) and two (HM 2) different grism settings. Because accurate guiding was not possible, the stars slowly drifted from the slit, and the resulting spectra (Fig. 1) are not absolutely flux-calibrated.
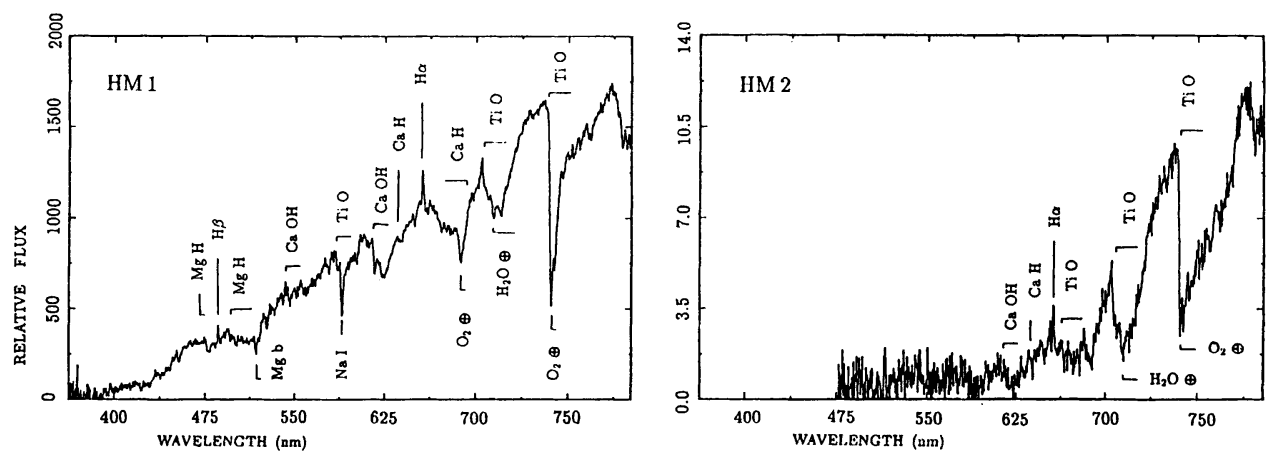

Fig. 1. Spectra of HM1 and HM2, observed with EFOSC 2 at the ESO $3.5 \mathrm{~m}$ NTT.

A spectral classification, using Turnshek et al.'s (1985) Atlas and Pettersen's and Hawley's (1989) spectral tracings, gave the results of Tab. 1 . In HM1, $\mathrm{H} \alpha$ and $\mathrm{H} \beta$ emission, in $\mathrm{HM} 2$, only $\mathrm{H} \alpha$ emission is visible.

With the spectral types and the photographic magnitudes of Hardy and Mendoza, the distances of HM1 and HM2 are found to be about $40 \mathrm{pc}$, much nearer than the distance of the $\mathrm{R} \mathrm{CrA}$ association. The stars are thus foreground objects or the $\mathrm{R} \mathrm{CrA}$ association reaches much nearer to us than previously assumed.

\section{Acknowledgements}

We thank W. C. Seitter for useful suggestions and comments. H.W.D. acknowledges support from the European Southern Observatory, and M.K.T. acknowledges support from the Alexander von Humboldt-Foundation.

\section{References}

Hardy, E., and Mendoza, E. (1968), Cordoba Bol. No.14, 28.

Marraco, H. G. and Rydgren, A.E. (1981), Astrophys. J. 86, 62.

Pettersen, B. R. and Hawley, S. L. (1989), Astron. Astrophys. 217, 187.

Turnshek, D. E., Turnshek, D. A., Craine, E. R. and Boeshaar, P. C. (1985), An Atlas of Digital Spectra of Cool Stars, Western Research Company, Tucson, Arizona. 